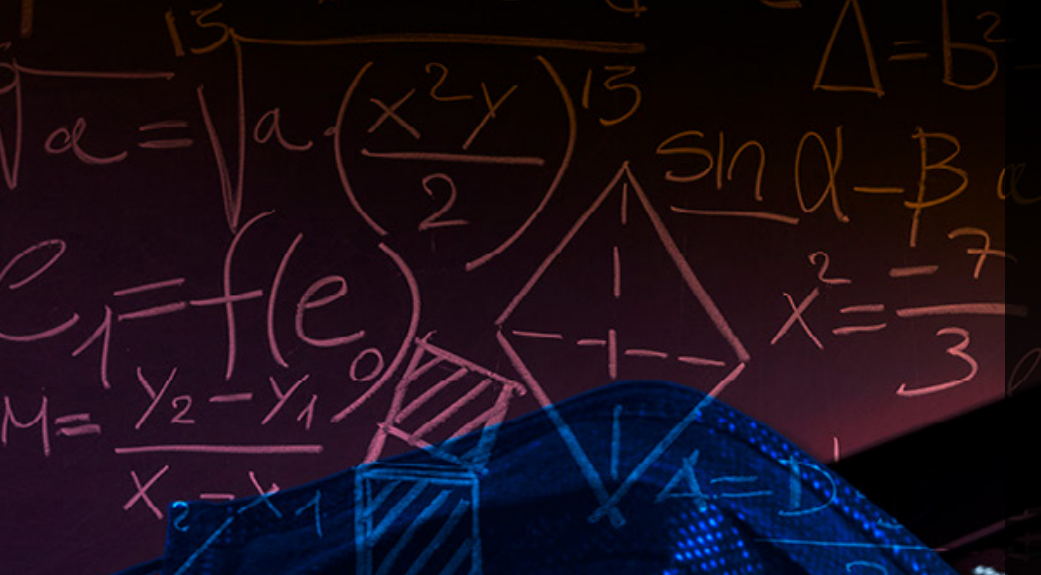

La pandemia del CovID-19, ha desatado una crisis que se expresa en varias dimensiones de la sociedad y que ha dejado ver, a todas luces, la desigualdad e inequidad social que existen en el ámbito de la educación superior en México. Las desigualdades e inequidades sociales se han visto exacerbadas frente al confinamiento obligatorio y la implementación de la modalidad de educación online "desde la casa", poniendo en evidencia la enorme brecha digital que existe entre IES, estudiantes y docentes. EI texto brinda elementos para reflexionar sobre este problema y acerca de algunas de sus posibles consecuencias.

Palabras clave: COVID-19, educación superior, desigualdad social, inequidad de género, educación online

\title{
COVID-19: \\ Efectos de la desigualdad social y la inequidad en la educación superior §IMéxico
}

\section{María Herlinda Suárez Zozaya ma_herlinda@yahoo.com.mx \\ Jorge Martínez Stack jorgestack@gmail.com}

\section{Introducción}

México destaca por ser un país en donde existe una enorme desigualdad social; esta situación se expresa en todos los ámbitos de la vida social y cultural, particularmente en el sistema educativo en todos los niveles. La desigualdad se encuentra relacionada con la distribución asimétrica de recursos materiales y simbólicos que guardan correspondencia con la estratificación social y en el ámbito de la educación se traduce, entre otros aspectos, en desigualdades de oportunidades de acceso, trayectorias y desempeño educativo.

Debido a la pandemia del coronavirus covid-19, los cursos presenciales están suspendidos. Estudiantes
Investigadora

del CRIM,

adscrita al

programa

Educación y

formación.

Miembro del

PUEES de la

UNAM

Asesor de

la Dirección

General de

Evaluación

Institucional, y

miembro del

PUEES de la

UNAM
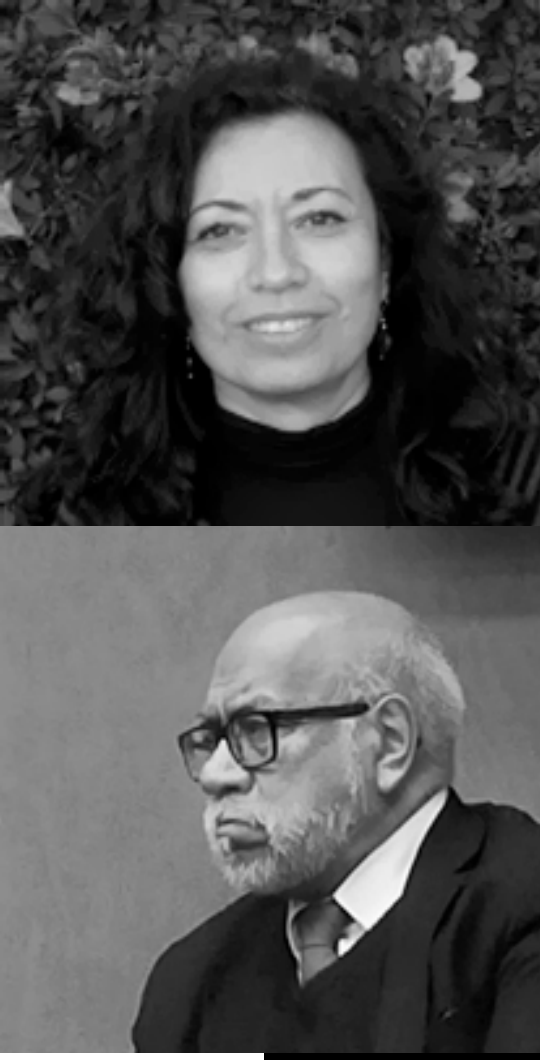
y profesores se encuentran sometidos al encierro en sus domicilios y algunas clases se imparten online. Este hecho ha expuesto con crudeza desigualdades e inequidades a las que, hasta ahora, el sistema educativo había prestado poca atención, debido a que refieren a carencias y condiciones de vida que suceden dentro de los hogares. El objetivo de este texto es brindar elementos para reflexionar sobre este problema y acerca de algunas posibles consecuencias en el ámbito de la educación superior.

\section{La estrategia de "Sana Distancia"}

En el mes de marzo, como medida precautoria para evitar contagios, las instituciones de educación superior (IES), públicas y privadas, suspendieron clases presenciales. La decisión se tomó con el objetivo de salvaguardar la seguridad y salud de los y las estudiantes y colaboradores; quedando enmarcada dentro de la estrategia nacional llamada: "Sana Distancia"1.

La Secretaría de Educación Pública (SEP) anunció que la estrategia permanecerá hasta el 30 de mayo, pero en el caso de los adultos mayores y personas con riesgo de complicaciones, se mantendrá hasta nuevo aviso. La verdad es que no está clara la fecha en la que las IEs reabrirán sus puertas, pero lo cierto es que el cierre de los establecimientos educativos resulta primordial para ralentizar la propagación del virus y evitar que los sistemas de salud colapsen. En México, como en muchos países del mundo, la cantidad de establecimientos y de personas involucrados en la educación superior es considerable. Según datos de la Subsecretaría de Educación Superior (sEs), la cantidad de personas que participa en el subsector es superior a los 5 millones y representa el 4.2 por ciento de la población nacional (Cuadro 1).

Cuadro 1: EUM. Educación superior

Cantidades de personas y planteles

\begin{tabular}{|c|r|}
\hline Total personas & $5,317,141$ \\
\hline Estudiantes & $4,700,000$ \\
\hline Docentes & 429,495 \\
\hline No docentes & 188,646 \\
\hline Total planteles & 6,404 \\
\hline IES públicas & 2,455 \\
\hline IES privadas & 3,949 \\
\hline
\end{tabular}

Fuente: elaboración propia con datos de Concheiro, 2020.

En un esfuerzo por no interrumpir los procesos formativos, algunas instituciones abrieron la opción de ofrecerlos en modalidad remota ${ }^{2}$. Para ello, pusieron a disposición de profesores y estudiantes, facilidades tecnológicas y soportes digitales; así, el proceso educativo se trasladó a los domicilios. La vivienda se convirtió en el espacio para el aprendizaje y, como era de esperar, en este escenario las desigualdades existentes se pusieron de manifiesto. 


\section{Oportunidad de acceso desigual}

Dejando de lado el problema de la baja cobertura que caracteriza a la educación superior en México, la oferta educativa de este nivel se encuentra altamente estratificada. Existen IEs con diferente dotación de recursos, de modalidades y prestigio. La heterogeneidad se da tanto entre escuelas públicas como privadas y las diferencias reproducen las desigualdades socioeconómicas del estudiantado.

ca

Entre los estudiantes matriculados en las IES, existen diferencias enormes respecto a la posibilidad

de cursar materias con el apoyo de herramientas

tecnológicas."

Entre los estudiantes matriculados en las IEs, existen diferencias enormes respecto a la posibilidad de cursar materias con el apoyo de herramientas tecnológicas. Varias universidades están equipadas para ofrecer educación a distancia, virtual y online; sin embargo, muchas no tienen las tecnologías de información y comunicación requeridas y carecen de la posibilidad de abrir canales de comunicación entre estudiantes y profesores fuera del aula. Al respecto, los resultados de la Encuesta Nacional de TIC, aplicada por la ANUIES, en 2019, muestran que el 39 por ciento de las IEs no of rece servicios académicos y de investigación mediante dispositivos móviles; el 22 por ciento lo hace, pero no de manera formalizada y sólo el 36 por ciento ofrece estos servicios de manera institucional (Gráfica 1).

Gráfica 1. eUm. Porcentaje de las IES que entregan servicios académicos y de investigación sobre dispositivos móviles

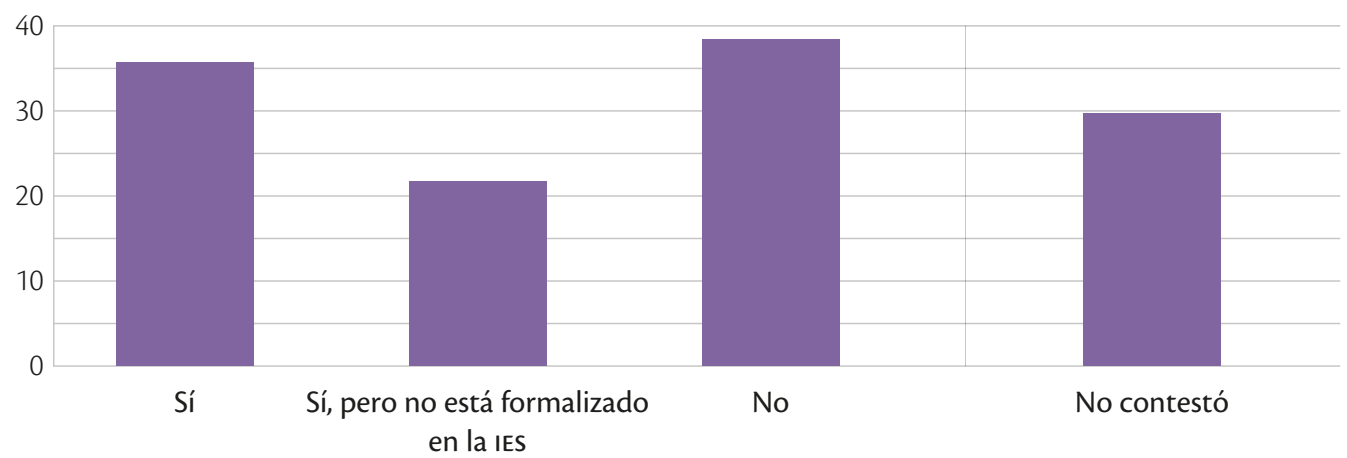

Fuente: Elaboración propia con información de anuies, 2019: 133

La distribución de las Tic y de las capacidades para su utilización, es muy desigual entre tipos de IES y también por campos disciplinarios, entre actores e individuos. Según estimaciones realizadas por la sEs, el 34.7 por ciento de la población estudiantil en IEs, públicas y privadas, no tiene computadora en su casa; el 27.6 por ciento no tiene conexión a internet y el 18.3 por ciento carece de ambos. Estos porcentajes se incrementan significativamente cuando se toma como referencia a los y las estudiantes pertenecientes al decil de ingreso corriente per cápita más bajo (decil I). Resulta que en este caso los porcentajes son: 61.5 por ciento, 77.5 por ciento y 53.7 por ciento, respectivamente. En cambio, para los y las estudiantes cuyos hogares se ubican en el decil más alto (decil X) los valores del indicador son: 6.6 por ciento, 14.6 por ciento y 3.2 por ciento, correspondientemente. En 
la Gráfica 2 se observa que la disponibilidad de dispositivos y de conexión se encuentra en relación directa con el nivel de ingresos.

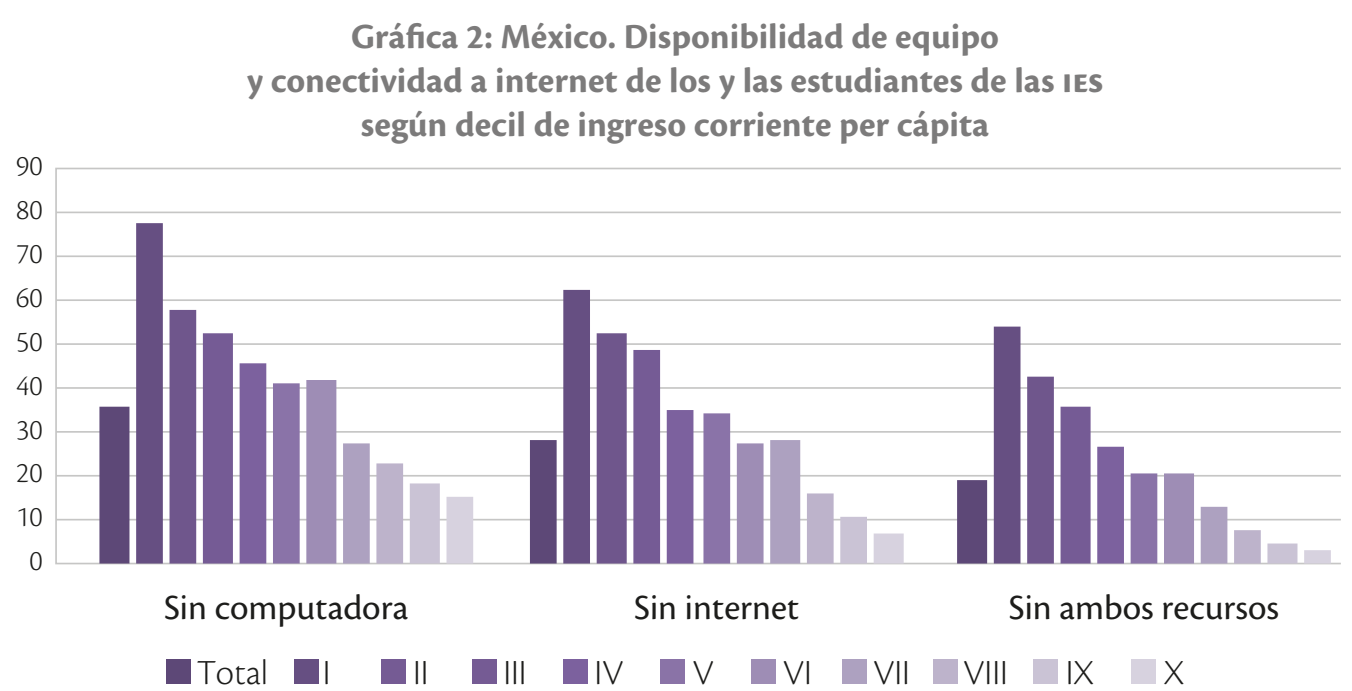

Fuente: Elaboración propia con base en los datos de Ramírez del Razo, Fuentes y Prieto, 2020.

Está claro: para una importante proporción de estudiantes de educación superior, continuar su proceso formativo desde casa está fuera de su alcance. En consecuencia, la estrategia de "Sana Distancia", en este nivel educativo, está operando como un factor potenciador de la desigualdad.

La manifestación de la desigualdad en la pandemia no solo refiere al acceso a dispositivos tecnológicos y a la conectividad de los y las estudiantes, también alude a la disponibilidad que tengan, de estos recursos, los y las docentes, así como de su capacitación para desarrollar estrategias y diseños pertinentes, que permitan poder desplegar transmisiones educativas en plataformas virtuales. Al respecto, Sahu (2020) señala las diferentes calidades de los cursos que se están impartiendo y además plantea las siguientes preguntas: ¿Qué pasa con los estudiantes cuyos cursos no pueden ser trasladados tan fácilmente a esta modalidad? ¿Cómo llevar a cabo prácticas, actividades de laboratorio o aprender música a distancia, cuando no se dispone de lugares y tiempos propicios o cuando no se cuenta con las competencias y las herramientas adecuadas?

Evidentemente estamos viviendo un momento inédito y particularmente difícil; es loable que a pesar del confinamiento exista posibilidad de seguir impartiendo y tomando cursos. Internet es una tecnología que puede ser democratizadora, pero en esta crisis ha emergido como un dispositivo que ha mostrado que, en el ámbito de la educación superior, como prácticamente en todos, el problema de desigualdad social es grave.

En el supuesto de que el cese de las actividades presenciales dure más de lo anunciado, lo probable es que la matrícula de educación superior caiga y que algunas ies privadas, cuyos ingresos dependen esencialmente del cobro de colegiaturas, tengan que hacer recortes de profesores e incluso habrá algunas que tendrán que cerrar. En efecto, es previsible que se produzcan deserciones e interrupciones en las trayectorias educativas y es de esperar que esto ocurra, en mayor medida, entre quienes provienen de los sectores sociales menos favorecidos, cuyos miembros han llegado a la educación superior en fechas relativamente 
recientes. Por lo tanto, es probable que entre los efectos concomitantes de la pandemia esté el del incremento de la exclusión social.

\section{Inequidad en las condiciones para el desempeño}

Los modos en los que los y las estudiantes y los y las docentes son socialmente construidos y se (auto) reconocen en sus hogares, no son coincidentes con los que operan en las escuelas. Sin olvidar que las personas forman grupos diferenciados y que tienen particularidades y especificidades propias, en general, la escuela representa un lugar de encuentro con pares, en el cual se producen dinámicas y diálogos en códigos académicos tanto en el salón de clases como fuera de estos. En cambio, en el espacio privado de la casa, en donde generalmente se convive con la familia, estudiantes y profesores suelen tener que responder a las prescripciones sociales asociadas a identidades, como la de hijo/a, esposo/a, madre/ padre, etcétera, que refuerzan la división de roles de la vida doméstica cotidiana.

La incidencia de los valores culturales en relación con el género, se da tanto en la escuela como en la casa, pero en los espacios familiares, las tradiciones y los valores heredados suelen actuar con mayor influencia sobre la orientación de los mandatos y las acciones de las personas. En estas circunstancias el proceso formativo de la educación superior, trasladado al ámbito doméstico, se ve perturbado por rutinas, actividades y tensiones propias del hogar, en donde persisten numerosos frentes de desigualdad; particularmente entre mujeres y hombres.

Gráfica 3. EUM. Prevalencia de las cuatro formas de violencia conyugal en el último año en mujeres unidas de $\mathbf{1 5}$ años y más que conviven con su pareja, según nivel de escolaridad de la mujer

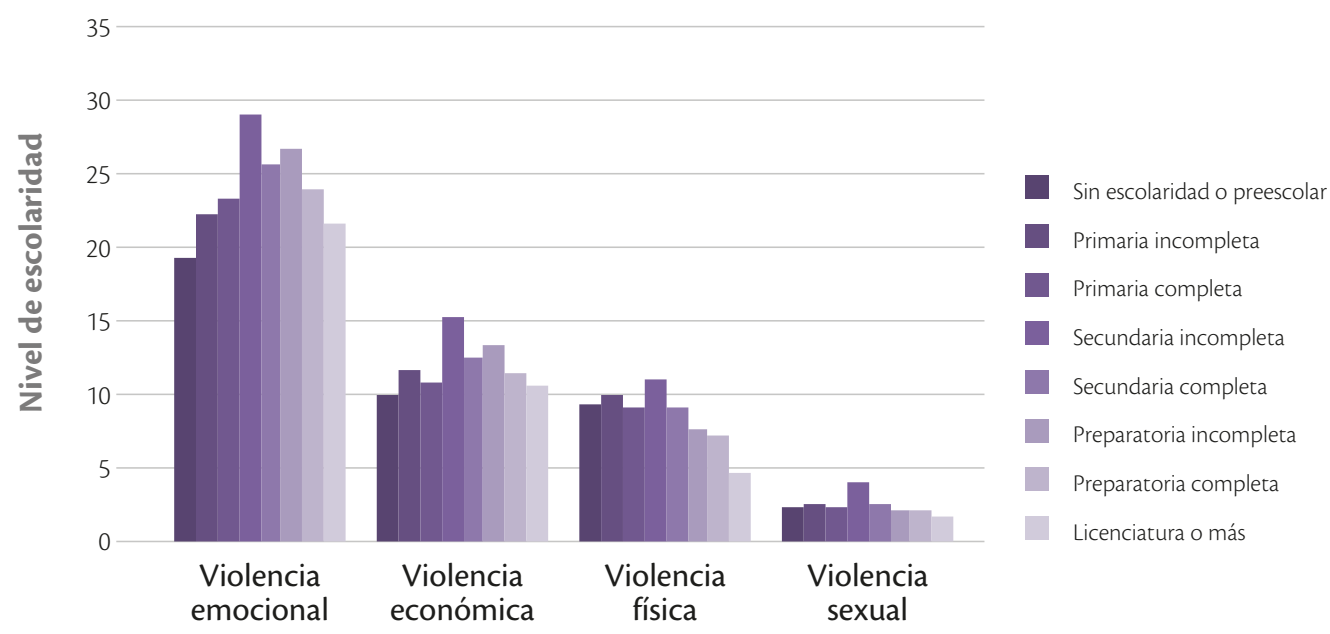

Tipo de violencia

Una investigación reciente reportada por el Fondo de Población de la Organización de las Naciones Unidas ha señalado que la violencia de género aumenta durante los periodos de encierro (UNFPA, 2020). Estima, que un impacto que se puede esperar de la 
crisis causada por el coronavirus, es que se incremente la violencia contra las mujeres y que incluso cause un retroceso en los avances que se habían logrado en términos de equidad de género ${ }^{3}$. En la gráfica 3 se observa que no está claro que para las mujeres, tener educación superior represente un factor de protección contra la violencia conyugal. $\mathrm{Si}$ bien las frecuencias de las violencias de tipo físico y sexual disminuyen (pero se siguen presentando), las de tipo emocional y económica aumentan; esto podría ser debido a que, con la escolaridad, la posibilidad de identificar este tipo de violencias se incrementa. Lo

\section{ca}

En este contexto, urge implementar

acciones concretas que eviten que solo algunos

tengan acceso y otros queden condenados

a una mayor precariedad." que es muy inquietante es que "las más altas prevalencias de violencias corresponden a aquellas mujeres que superan a sus parejas en términos de años de escolaridad" (Casique, y Castro, 2019, p: 178).

Ahora bien, según datos de Encuesta Nacional de Ingresos y Gastos en los Hogares (2018), más de la mitad de la matrícula de educación superior en México, está compuesta por mujeres (51 por ciento). Por su parte, la cobertura por sexo es casi idéntica ( 37.7 por ciento y 37.6 por ciento) y, en este nivel educativo, las mujeres suelen tener mejor rendimiento escolar que sus compañeros varones. Sin embargo, la estrategia de Sana Distancia, que ha convertido la casa en el espacio para el estudio y el aprendizaje, ha implicado que prácticamente todos los integrantes estén teniendo que dedicar mayor tiempo a las tareas domésticas; pero la mayor carga está siendo para las mujeres, sean estudiantes o profesoras. Varias están llevando cursos desde sus hogares y prácticamente al mismo tiempo deben atender necesidades y demandas domésticas. La carga excesiva de trabajo y lo inadecuadas que resultan las condiciones de arraigo domiciliario, dificultan poder atender cabalmente las trasmisiones educativas. De ahí que, entre los efectos de la pandemia se encuentre la inequidad de oportunidades de desempeño, según género.

Es imposible identificar la totalidad de las implicaciones que el coronavirus tendrá en la educación superior. Por lo pronto no hay que olvidar que en la actualidad proliferan las empresas que aprovechan toda oportunidad para expandir sus fortunas y que es de esperar que la oferta de educación virtual, el mercado de plataformas para esta modalidad, el consumo de dispositivos tecnológicos y las ofertas de telecomunicación para conectarse a internet, se multipliquen. En este contexto, urge implementar acciones concretas que eviten que solo algunos tengan acceso y otros queden condenados a una mayor precariedad.

Durante la pandemia, la estrategia de "Sana Distancia", ha revelado que la propuesta de educación superior universal, contemplada como derecho en la Constitución Mexicana, no podrá hacerse realidad si no se presta atención a las causas que subyacen a las desigualdades e inequidades en materia educativa, las cuales están configuradas por el entorno en que vive la población y por el modo de vida. Es momento de pensar las metas educativas con perspectiva histórica y admitir que las carencias, la desigualdad y las inequidades que sufren millones de personas en México, representan una amenaza para los logros sociales conquistados. 


\section{Notas}

1 El 16 de marzo de 2020, la Secretaría de Salud anunció la implementación de la Jornada Nacional de Sana Distancia como medida preventiva. De esta manera, se suspendieron temporalmente las actividades no esenciales, se reprogramaron los eventos de concentración masiva. Igualmente, se adelantó el periodo vacacional de Semana Santa en las escuelas. En un principio se estableció que esta Jornada Nacional de Sana Distancia concluiría el 19 de abril, fecha que se postergó más tarde hasta el 30 de mayo.

2 Es importante aclarar que la educación a distancia, la educación virtual y la educación online no son términos intercambiables. Durante la pandemia, lo que está sustituyendo a los cursos presenciales es educación online, cuya característica es que ocurre en tiempo real. En esta modalidad las clases y las reuniones se programan en horarios precisos y se emiten en directo de forma telemática. Aunque en realidad, la continuidad de las clases depende de cada docente, en función de cómo se organiza cada uno.

3 Datos proporcionados por el Secretariado Ejecutivo del Sistema Nacional de Seguridad Pública señalan que "la violencia de género ha alcanzado niveles nunca vistos durante el periodo de confinamiento.”(De Mauleón, 2020)

\section{Referencias bibliográficas}

Ponce, J. L. (coord.) (2019). Estado actual de las Tecnologias de la Información y la Comunicación en las Instituciones de Educación Superior de México: Estudio 2019. México: ANuies. estudio-tic.anuies.mx

Casique, I. y Castro, R. (2019). "Cambios y constantes en los niveles y factores asociados a las violencias de parejas en México”. En Castro, R. (coord.), De parejas, hogares, instituciones y espacios comunitarios. Violencias contra las mujeres en México (Endireh, 2016) (pp.161-270). INMUJERES, CRIM-UNAM.

Concheiro, L. (17 de abril de 2020). "Respuestas de las instituciones públicas de educación superior en México para enfrentar la crisis del covid-19.” Reunión de trabajo llevada a cabo en la Asociación Nacional de Universidades e Instituciones de Educación Superior, Cd. De México, México.

De Mauleón, H. (2020). Señor presidente: hay otros datos. El Universal. Recuperado el 7 de mayo de 2020 en: https://www.eluniversal.com.mx/opinion/hector-de -mauleon/senor-presidente-hay-otros-datos

Encuesta Nacional de Ingresos y Gastos de los Hogares (2018). Nueva serie. Recuperado en: https://www.inegi.org.mx/programas/enigh/nc/2018/default. html\#Datos_abiertos

Ramírez, H., Fuentes, C., Prieto, B. (2020). Estimaciones de los estudiantes de educación superior, media superior, educación secundaria y educación primaria, que carecen de dispositivos de cómputo y conexión a internet en sus hogares, por decil de ingreso corriente per capita. Estimaciones elaboradas por la SES-SEP. Con base en inegi. Encuesta de Ingresos y Gastos de los Hogares, 2018. Documento de trabajo.

Sahu, P. (4 de abril de 2020). Closure of Universities Due to Coronavirus Disease 2019 (COVID-19): Impact on Education and Mental Health of Students and Academic Staff. 
Cureus 12(4): e7541. doi:10.7759/cureus.7541. Recuperado en: https://www. cureus.com/articles/30110-closure-of-universities-due-to-coronavirus-disease2019-covid-19-impact-on-education-and-mental-health-of-students-andacademic-staff

Fondo de Población de las Naciones Unidas. (2020). Comunicado del unfpa sobre el nuevo coronavirus (COVID-19) y el embarazo. Recuperado el 5 de marzo de 2020, en: https://www.unfpa.org/es/press/comunicado-del-unfpa-sobre-el-nuevocoronavirus-covid-19-y-el-embarazo 